\title{
Natural Ventilation in Urban Buses: From Negative Pressure To Velocity-Driven Dispersion of Aerosols
}

\section{F. Alexei Pichardo-Orta}

Autonomous University of San Luis Potosí

Oscar Adrían Patiño-Luna

Autonomous University of San Luis Potosí

J. Rodrigo Vélez-Cordero ( $\nabla$ jivelez@ifisica.uaslp.mx )

Autonomous University of San Luis Potosí

\section{Research Article}

Keywords:

Posted Date: February 15th, 2022

DOI: https://doi.org/10.21203/rs.3.rs-1308008/v1

License: (1) This work is licensed under a Creative Commons Attribution 4.0 International License.

Read Full License 


\title{
Natural ventilation in urban buses: from negative pressure to velocity-driven dispersion of aerosols
}

\author{
F. Alexei Pichardo-Orta ${ }^{1}$, Oscar Adrián Patiño Luna ${ }^{1}$, and J. Rodrigo Vélez Cordero,"* \\ ${ }^{1}$ Instituto de Física, Universidad Autónoma de San Luis Potosí, Álvaro Obregón 64, 78000 San Luis Potosí, S.L.P., \\ México \\ ${ }^{2}$ Investigadores por México-Instituto de Física, Universidad Autónoma de San Luis Potosí, Álvaro Obregón 64, \\ 78000 San Luis Potosí, S.L.P., México \\ *jrvelez@ifisica.uaslp.mx
}

\begin{abstract}
In this report we analyze the air streams formed across the open windows (natural ventilation) of an urban bus model and the resulted dispersion of aerosols emitted in the passenger area. The methods include computational fluid dynamics simulations and three ways to characterize the dispersion of passive tracers: a continuous concentration-based model, a discrete random model and a parametric scalar based on the so-called mean age of air. We also conducted experiments using a 1:10 scale bus model and $\mathrm{CO}_{2}$ as a passive tracer to assess the ventilation characteristics. We found that dispersion and expulsion of aerosols is driven by a negative pressure field in the standard bus design equipped with lateral windows. Also, the average age of air is 6 minutes while the air streams promote aerosol accumulation at the front (driver's area). To speed up the expulsion of aerosols and reduce their in-cabin accumulation, we proposed a bus bodywork prototype having a frontal intake of air. The expulsion of aerosols in this case is driven by a frontal velocity field while the average age of air is reduced to 50 seconds which manifests as an increase of the aerosols expulsion rate.
\end{abstract}

\section{Introduction}

Covid-19 pandemic has motivated research groups around the world to intensify investigations on airborne pollutants. Although definite conclusions have still wait for more rigorous and controlled experiments ${ }^{1,2}$, an increased amount of evidence and study cases have stressed out the importance of airborne transmission and residence times of virion-containing droplet nuclei in the propagation of the pandemic, ${ }^{3-6}$ particularly in places with little security such as confined, crowded or poorly ventilated areas $^{1,7}$ (for transmission cases in the public transport see Refs. ${ }^{3,8}$, also see Ref. ${ }^{9}$ for an aerosol transmission experiment using animal models). A general call out has therefore been given to move or prefer activities outdoors, if possible, ${ }^{10,11}$ together with the regular practice of other security measures such as frequent ventilation, ${ }^{12}$ mask wearing or the practice of physical distancing. The goal of this report is to offer general guidelines and purpose novel designs to improve the ventilation and expulsion rate of aerosols emitted inside urban buses; in particular, these guidelines can be used in emergencies as the one we are currently living.

Studies on turbulent flows inside urban buses, together with the flow-induced propagation of airborne species, have already been conducted by several research groups using computational fluid dynamics (CFD). In a first group of papers we can find those that considered turbulent flows inside a bus generated by an air conditioning system ${ }^{8,13-15}$ In this situation, the position of air entries and exhausts together with the amount of recirculated air or amount of fresh air added to the mixture can be considered as design variables to test different ventilation modes and improve the expulsion of internal emitted aerosols. There are other papers which, on the other hand, have simulated in-cabin turbulent flows at the expenses of the exterior air flowing through open windows (having no air conditioning systems, as in Latin American, Asian and African countries); these papers $^{8,16-19}$ conform the main background of the present report. First of all, the majority of these studies have revealed a characteristic flow inside urban buses that may not be obvious at first glance: contrary to what we may expect, when a bus is moving at a certain speed having a fixed number of open windows, the exterior air flow is observed to enter from the back windows and then travels towards the front pushing or sweeping the aerosols from back to front, on average. This counterintuitive flow happens because pressure is lower at the front windows compared to the values at the back, causing this pressure driven flow. Another important observation taken from these studies is the fact that, perhaps not surprising, removal of indoor particles is accelerated when the bus windows are maintained open. ${ }^{8}$ In particular, Li and coworkers ${ }^{16}$ studied the flow characteristics and drag of contaminants generated by different arrangements of open windows; notoriously, they remarked that opening the driver's window and windows located at the middle of the bus can led to an observable "pumping effect" which 
transport air form back to front. Li's papers as well as other works ${ }^{16,20,21}$ have focused mainly on the transport of pollutants generated outside the bus, such as engine exhaust gases, which can then infiltrate to the interior of the bus, or on the temperature distribution inside the bus generated by the flow field and its impact on thermal comfort levels. ${ }^{17,19}$ Only Zhang $^{8}$ have explicitly considered the problem of aerosols emitted and transported inside a bus but without following its expulsion to the exterior (also consult the work of Mesgarpour ${ }^{22}$ on the spread of droplets inside a closed bus). It is therefore necessary to conduct a new study on the transport and expulsion of aerosols occurring across the open windows of an urban bus by solving the external and internal flows simultaneously.

It is important to mention that ventilation quality or removal of pollutants from indoor spaces have also been studied computationally in other kind of public spaces; relevant examples include the flow characteristics and motion of airborne species inside an aircraft cabin ${ }^{23,24}$, the flow inside a high speed train cabin ${ }^{25}$ and inside an automobile ${ }^{26}$ (these authors also noticed the aforementioned back-to-front pumping effect); flow and aerosol dispersion inside a conference room, ${ }^{27}$ inside a generic ventilated room ${ }^{28}$, a supermarket ${ }^{29}$ or a restaurant ${ }^{30}$, and the flow inside buildings and hospitals (see Ref. ${ }^{31}$ for a general review on the subject).

\section{Results}

An image of a typical urban bus is shown in Figure 1a while Figures $1 \mathrm{~b}$ and 1c show the numerical and experimental models used in this report.
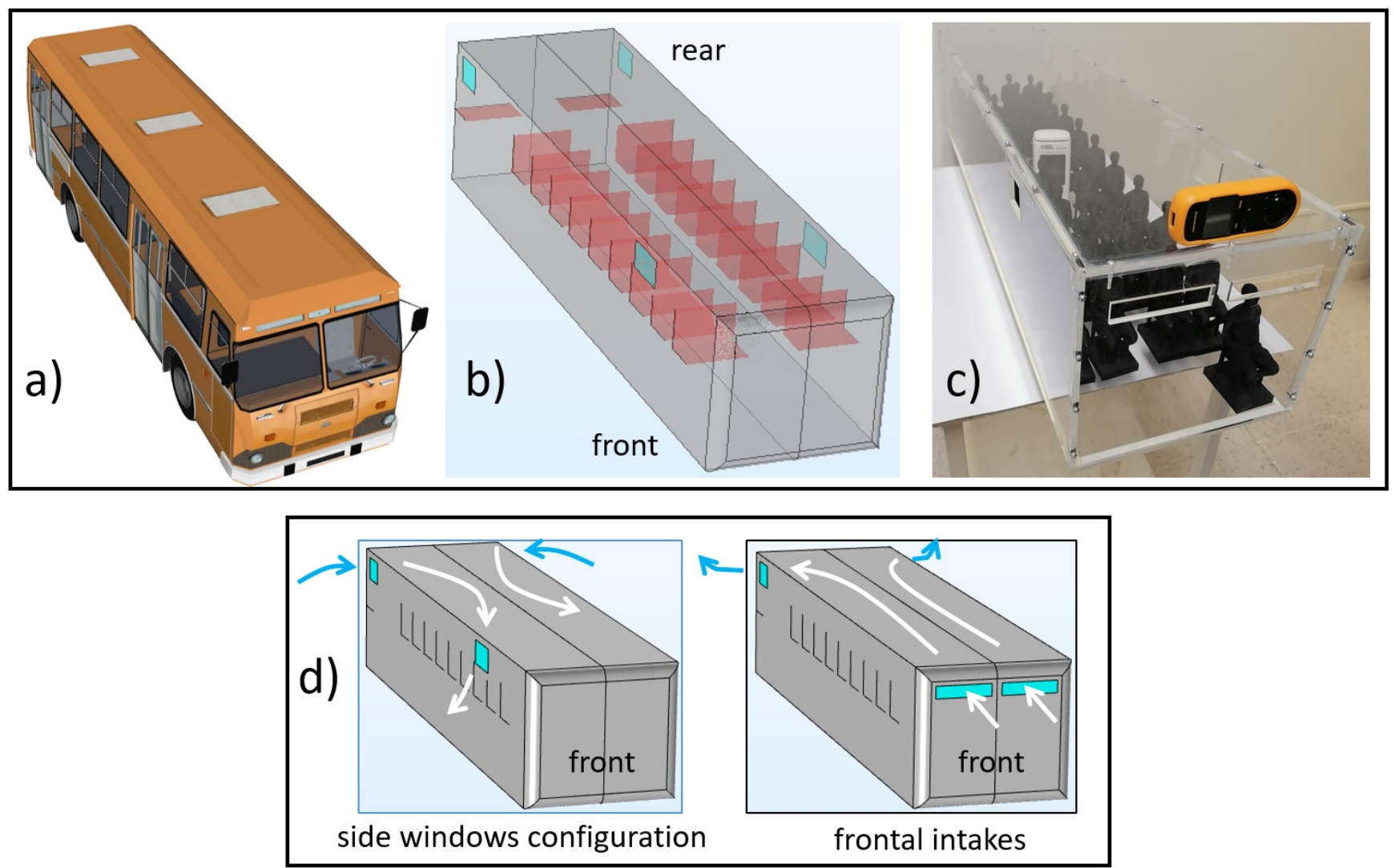

Figure 1. Image of an urban bus (a) together with an overview of the numerical (b) and experimental (c) models used in this study (for more details see the Methods section). Figure (d) shows a scheme of the air streams direction observed in the lateral windows configuration in which air is pumped from back to front, as well as the proposed configuration in which air intakes are installed at the frontal wall, changing the mean flow direction. In the numerical model (b) seats are denoted with red color while open windows are marked in blue.

Briefly (the details can be consulted in the Methods), the numerical model solves for the internal and external air flow formed along a bus traveling at a representative speed of $U=50 \mathrm{~km} / \mathrm{h}(13.9 \mathrm{~m} / \mathrm{s})$ and having a certain distribution of open 
windows. An example of what happens at lower speeds are presented in the supplementary material S1. Once we got the turbulent air flow field we proceeded to evaluate the ventilation of internal emitted aerosols in three different ways. In a first approach we analyze the drag and expulsion of a continuous "cloud" of particles (concentration field) emitted by a Gaussian pulse having 0.5 seconds width and located at the center of the passenger area. To get a more realistic simulation of a breathing (exhalation) event, we also modeled the aerosols as a statistical ensemble of Lagrangian particles using a set of random parameters (particle velocity) in order to account for the stochastic nature of the aerosol release. Finally, since the ventilation characteristics of these Eulerian and Lagrangian representations depend on the the emission site itself, we also computed the so-called mean age of air for a given flow field to visualize the re-change of indoor air. By definition, this parameter reports how difficult is for a given emission exit a control volume for any possible location of the emitter.

To be able to assess experimentally the ventilation characteristics inside the bus and explore new air entries locations; i.e., explore non-existent distributions of open windows, we used an acrylic 1:10 scale bus model having 3D-printed passenger figures as our experimental setup (see Fig. 1c). As the scaling parameter we chose to preserve the time it takes the air to travel the full bus length, i.e., $t=L / U$, where $L$ is the length of a real sized bus $(\sim 10 \mathrm{~m})$ or the length of our scaled model ( $1 \mathrm{~m})$, while $U$ is the traveling speed of a real bus $(13.9 \mathrm{~m} / \mathrm{s})$ or the speed of our scaled bus $(1.3 \mathrm{~m} / \mathrm{s})$. Notice that this scaling option will reduce the Reynolds number by a factor of 100 but will, nevertheless, maintain the turbulent regime (from $2 \times 10^{6}$ to $2 \times 10^{4}$ in the model). Additionally, we used a pulse of $\mathrm{CO}_{2}$ and a commercial sensor to evaluate the ventilation characteristics in the experimental model given a certain arrangement of open windows.

Now we want to comment on the main hypothesis of this report and which is illustrated in Figure 1d. In the current commercial bus designs, the windows are placed along the lateral walls of the bodywork, typically 4 to 5 sliding windows on each side. As mentioned in the introduction, this lateral arrangement promotes inner ventilation or particle sweeping from back to front, on average, due to the lower pressure front established at the wall of attack. The new configuration we want to explore is depicted on the right image of Figure 1d and consist in collocating two open windows or air entries at the front wall while leaving two lateral windows open at the back. We hypothesize that this configuration will not only change the average ventilation direction (front to back) but will also speed up the expulsion of internal emitted aerosols. Although these new air entries proposed here do not exist in commercial vehicles, it is worth saying that the current bus designs have available space in the area between the driver's windshield and the bus roof (usually used to announce the bus route). It is this space where we put the two frontal windows either in the computational or experimental models.

\section{D simulations: a first exploration}

We stated exploring the effects of the different open windows configurations by conducting 2D simulations on a horizontal plane coincident with the height at which the windows are located. These simulations also helped us to optimize the mesh size and compare the $\kappa-\varepsilon$ turbulent model with the more rigorous, but demanding, SST $\kappa-\omega$ model (the details can be consulted in the Methods and in the supplementary material S2, S3 and S4, including validation examples of the SST $\kappa-\omega$ model). The different distributions of open windows that we studied are depicted in Figure 2 and have the following nomenclature: $2 \mathrm{~W}$ for 2 open windows, $4 \mathrm{~W}$ for 4 open windows, AW for all windows open, and FW for the new proposed arrangement consisting in opening two air entries at the front wall together with 2 lateral windows at the back. Additionally, in these $2 \mathrm{D}$ simulations we used the concentration-based model for the aerosols, choosing the source of emission to be at the middle of the bus (marked with asterisks in the figure). The plot shows the total amount of aerosols, normalized with the corresponding maximum values or peak values, being inside the bus at any time after the $0.5 \mathrm{~s}$ emission pulse (the Gaussian tail starts at time $t=0$ ). Blue lines are the results using the $\kappa-\varepsilon$ model while the yellow lines correspond to the SST $\kappa-\omega$ model.

At first glance we can see that the rate of expulsion of aerosols increases as we go from 2 to 4 open windows, as is expected, while opening all the windows do not makes much difference with respect to $4 \mathrm{~W}$ (we have included some movies in the supplement so that the reader can have a clearer picture of the dispersion characteristics). The $2 \mathrm{~W}$ distribution permits the expulsion of aerosols even though it does not promote the establishment of the back-to-front pumping effect or air circulation since the back windows are close. The observed lag time to start expulsion, $\sim 10 \mathrm{~s}$, is because the inner air streams are so slow that the aerosol cloud takes some time to reach the open windows location. The $4 \mathrm{~W}$ and AW configurations show, on the other hand, a notable improvement of the expulsion rate because they speed up the inner currents due to the establishment of the pumping effect. Therefore, it is very important for a proper circulation and renewal of air to leave at least two open windows at each lateral wall, two at the front and two at the back. In the supplementary material S4 we have included some fluid flow maps to see this pumping effect. Finally, we can see that the FW configuration displays the best performance in terms of the expulsion rate; this gave us confidence to continue with the $3 \mathrm{D}$ simulations and experimental tests.

\section{D simulations: flow field and age of air}

In the 3D simulations we analyzed with more detail the lateral $4 \mathrm{~W}$ and frontal FW configurations. Figure 3 shows some 3D-streamlines (black lines) together with a representative pressure field map, as well as the mean age of air for these two open windows distributions in an unoccupied bus. From figures $3 \mathrm{a}$ and $3 \mathrm{c}$ it is clear that while the pressure field at the windows level 


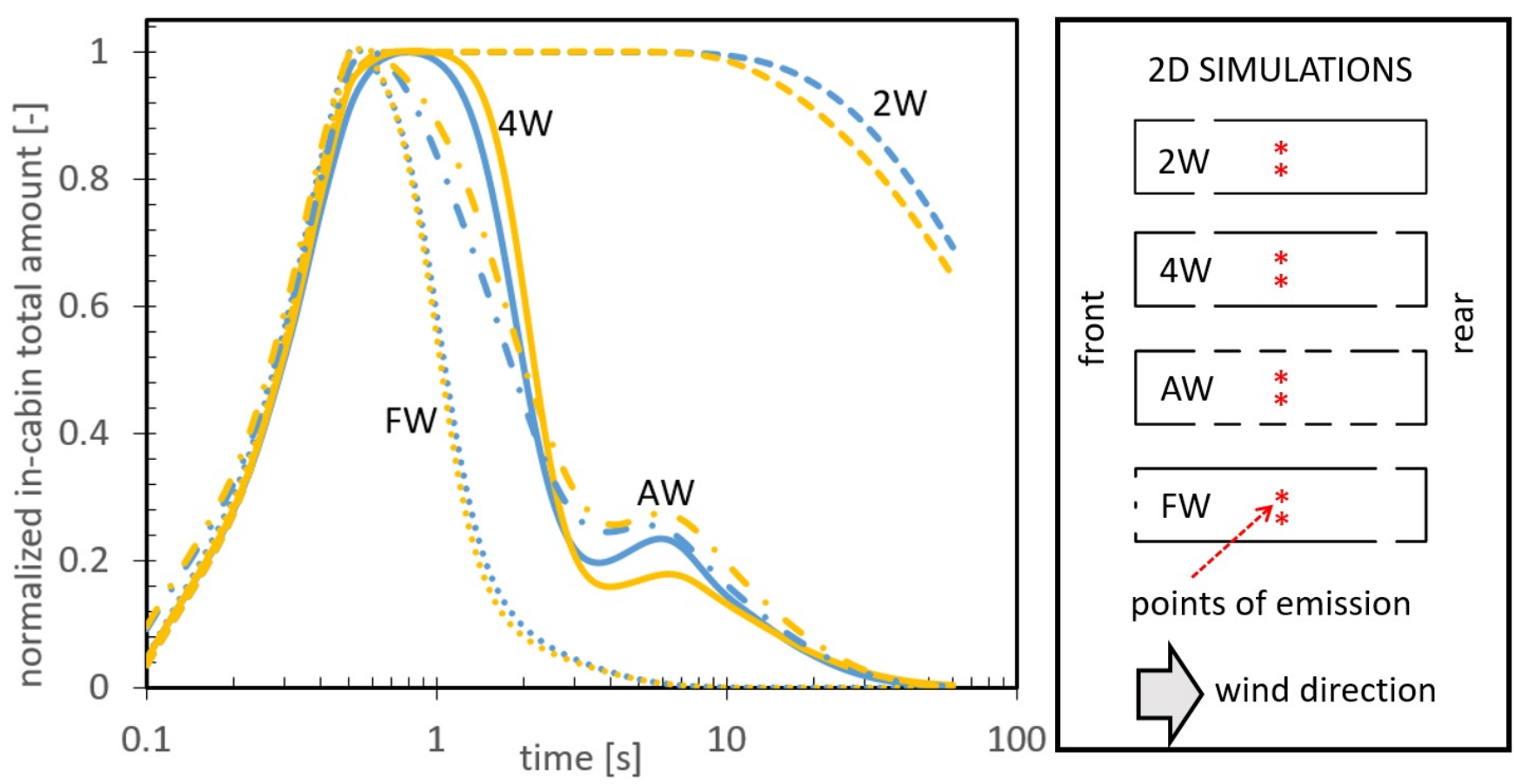

Figure 2. 2D simulations: total amount of aerosols inside the bus as a function of time normalized with the corresponding maximum values. Blue lines are the results using the $\kappa-\varepsilon$ model while yellow lines correspond to the SST $\kappa-\omega$ model. The explanation of the different open windows configurations are explained in the text and in the scheme at the right side. Aerosol emission occurs in the first 0.5 seconds at the center of the bus cabin. See also the movies included in the supplementary material.

is similar in both situations outside the bus, the pressure sign in the cabin changes from negative (suction effect) in the $4 \mathrm{~W}$ case to positive (thrust effect) in the FW arrangement. The air streamlines also have a different destiny in each case: in the 4W configuration the streamlines coming from the front circumvent the bus bodywork without entering the cabin; in contrast, in the FW case they enter the bus through the frontal entries, traveling the bus interior and exiting through the back windows.

The corresponding 3D-contour plots of the mean age of air are shown in Figures $3 \mathrm{~b}$ and $3 \mathrm{~d}$ (a validation plot for the mean age of air, including its values inside and outside the bus cabin, is shown in the supplementary material S5). The age of air or average re-change time in the $4 \mathrm{~W}$ case has an average value of $378 \mathrm{~s}$ ( $\sim 6$ minutes) with a maximum of 7.8 minutes appearing mostly at the front. In the FW configuration the mean age of air was 50s with a maximum of 69s. Among all the numerical results this is perhaps the most outstanding difference between the negative pressure-driven flow found in $4 \mathrm{~W}$ and the proposed FW configuration having the thrust effect.

\section{Statistical ensemble of aerosols}

To obtain a more realistic simulation of the dissemination and expulsion of aerosols emitted inside the bus, in this section we show the results of following a statistical ensemble of discrete particles transported by the background flow using random parameters in order to capture the stochasticity of an exhalation event (please consult the methods for a more detailed explanation of the release protocol). The exhalation event consist in releasing 100 particles during a time period of $2 \mathrm{~s}$ and then follow their trajectories as a function of time; this event is replicated 40 times each time changing the release parameters randomly to have a total ensemble population of 4000 particles. The release site was the same as that chosen for the concentration-based model.

An example of an exhalation event is shown in Figure $4 \mathrm{a}$ where we can see the particle bundle at 6 s after emission, coming out at seat number 6 , and the same particles but now at time $46.5 \mathrm{~s}$ (see how some particles start leaving the bus through the back window). Figures $4 \mathrm{~b}$ and $4 \mathrm{c}$ show the number of particles inside the bus at any time for the $4 \mathrm{~W}$ and FW configurations; the gray lines represent each replica of the ensemble while the red line is the ensemble average. We have also included in the insets the $\operatorname{pdf}(\mathrm{y})$ (probability density function) of finding the particles at the last time of the simulations in terms of the longitudinal distance (release site is at $\mathrm{y}=5 \mathrm{~m}$ or seat \#6). It is important to say that we imposed the sticky condition to the particles so that they become immobilized whenever they touch a solid surface.

For the $4 \mathrm{~W}$ case (Fig. 4b) we can see that after a retention time, the particles start exiting the cabin through the open windows at an average rate of 2 particles/minute. On the other hand, the pdf(y) shows a higher probability to find the particles 

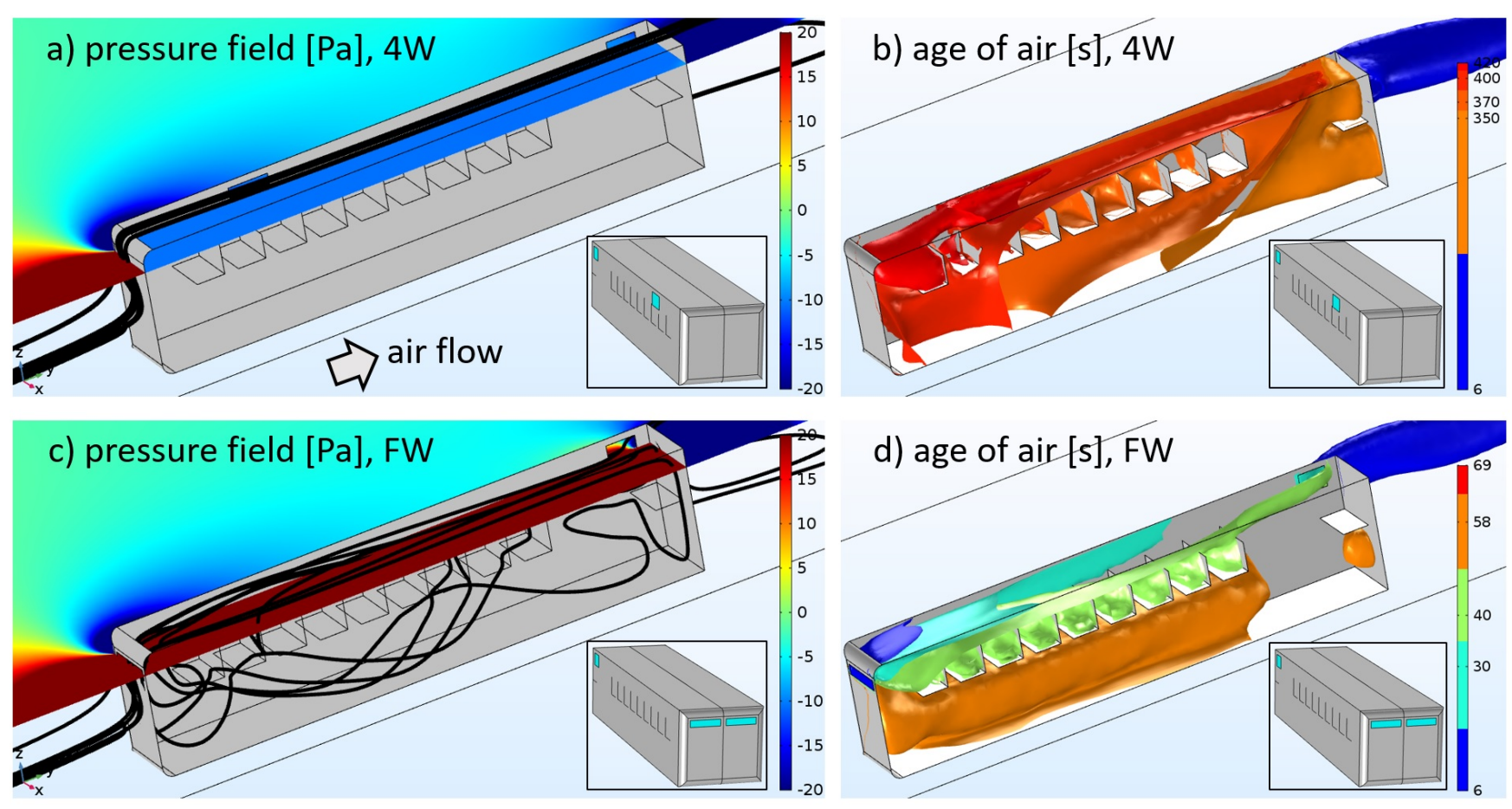

Figure 3. 3D simulations: pressure field, streamlines (black lines) and mean age of air for the $4 \mathrm{~W}$ and $\mathrm{FW}$ configurations (see also the insets as a guide). Air flow direction is indicated with an arrow; the roof of the bus is not shown just for easy viewing inside.

near the release site and in the front side. Particle expulsion is different in the FW case (Fig. 4c) because the retention time is followed by a rapid increase of the expulsion rate, $\sim 28$ particles/minute, but then decreases and reaches a stationary value. The corresponding $\operatorname{pdf}(\mathrm{y})$ shows that the localization probability is more narrow compared to the $4 \mathrm{~W}$ case and laden to the rear side. It is important to remark that the plateau observed in the main plot is due to the fact that almost all the particles that have not left the bus at early times become arrested at the solid inner walls. In the same sense, the continuous decrease observed in the $4 \mathrm{~W}$ case reveals that the majority of the particles stay suspended in air. In summary, all this data indicate that the aerosol expulsion in the lateral $4 \mathrm{~W}$ case is slow, continuous and maintain aerosols suspended in air for longer times than the FW configuration where the expulsion is faster, reach a steady state and promotes particle settling.

\section{Experimental validation}

All the numerical simulations were done taking into account the basic bus design and distribution of open windows, i.e., an unoccupied bus. In this section we present experimental results considering a situation in which the bus is fully occupied (see Figs. 1c and 6c, d). As described in detail in the Methods, for the experiments we used a $0.5 \mathrm{~s}$ pulse of $\mathrm{CO}_{2}$ released at the middle of the bus model and then register its concentration decay at the front (driver's area), middle and back of the bus using a commercial $\mathrm{CO}_{2}$ sensor. Figures $5 \mathrm{a}, 5 \mathrm{~b}$ and $5 \mathrm{c}$ show the normalized $\mathrm{CO}_{2}$ amount for these three positions and for the 4W and FW cases. The rate of decay or expulsion of $\mathrm{CO}_{2}$ is, in all cases, higher in the FW case but the difference is particularly notorious in the driver's area. Figure 5d shows the maximum absolute value (ppm) of $\mathrm{CO}_{2}$ detected after release for the three positions and the two configurations. Standout the fact that the maximum amounts are seen for the driver's and middle positions in the $4 \mathrm{~W}$ case, although at the back the maximum amount is higher for the FW case. In the supplementary material S6 we have included more experimental data including cases where only 2 windows are open and a control where none of the windows are open.

\section{Discussion}

The present study was inspired on an emergency situation in which small airborne hazard particles, or aerosols, are emitted inside an urban bus and have to be expelled out in the shortest possible time (maximum expulsion rates). In the context of the current emergency brought by COVID-19, the study of ventilation efficacy in the public transportation is highly pertinent since studies indicate ${ }^{32,33}$ that the highest risk of infection is seen in urban buses compared to other modes of transportation. 

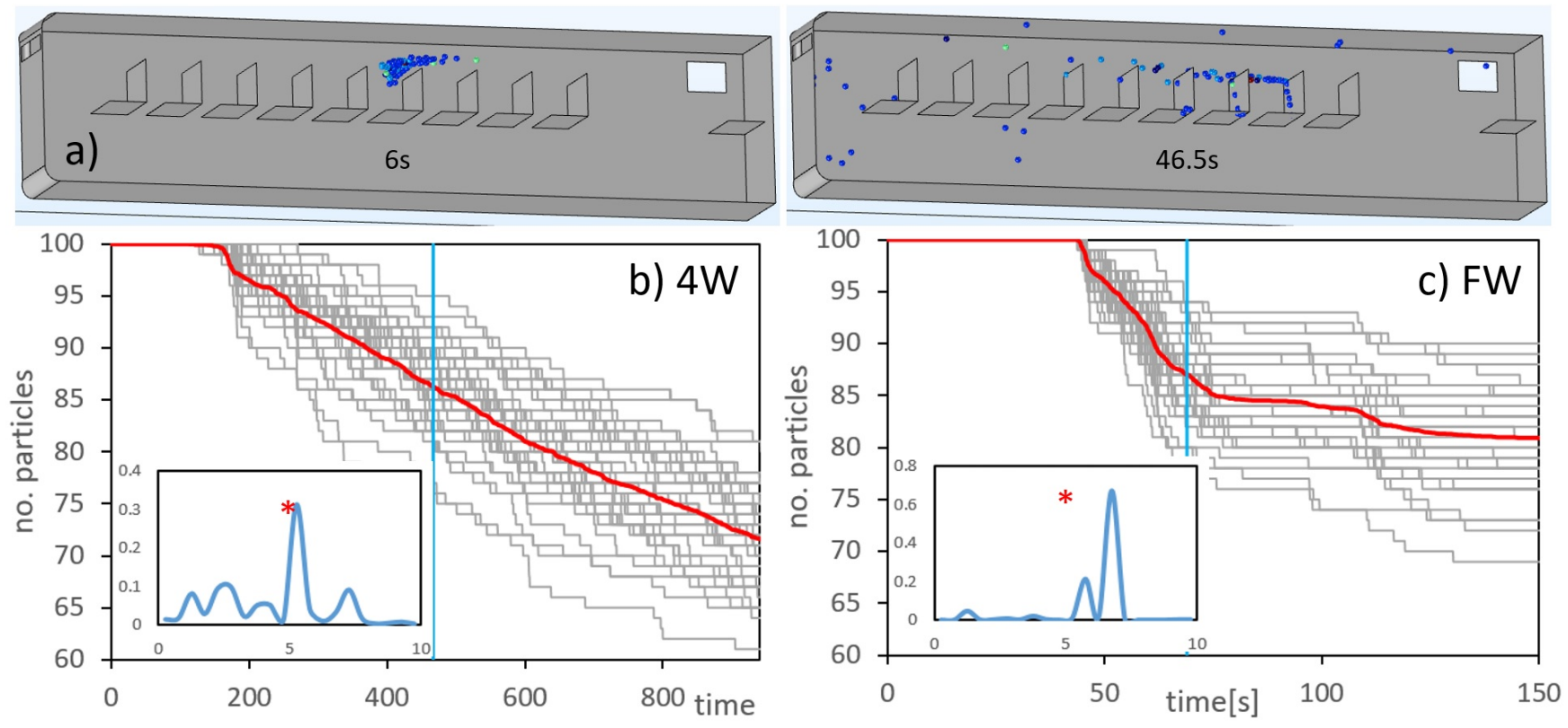

Figure 4. 3D particle tracking; (a) example of the position of 100 particles 6 and 46.5s after emission; (b) and (c): number of particles inside the bus as a function of time (seconds) for every exhalation event (gray lines) and for the ensemble average (red line). The vertical blue line in the main plots indicates the average age of air values for the $4 \mathrm{~W}$ and $\mathrm{FW}$ cases; the insets show the $\operatorname{pdf}(\mathrm{y})$ of the particle position at the last time of the simulation, the asterisks indicate the position of the particles release.

The key message and proposal of this report was that the installation of front-upper casement windows on the bus bodywork can substantially improve the ventilation quality in comparison with current designs in which air entries are exclusively distributed along the lateral walls of the bus. We have shown with experiments and numerical simulations that the frontal FW configuration proposed here increases the expulsion rate of aerosols while reducing the maximum amount of retained species after release; it also reduces the particle spreading inside the bus and the mean age of air by one order of magnitude with respect to the standard open windows configurations. As an additional note, the total flow rate in the FW configuration with the bus moving at $50 \mathrm{~km} / \mathrm{h}$ and having an occupation of 50 people will yield a value of $\sim 100 \mathrm{~L} / \mathrm{s} /$ person, a value that is well above the recommended ventilation rate $\left(8-10 \mathrm{~L} / \mathrm{s} /\right.$ person) proposed by the Scientific Advisory Group of Emergencies (SAGE) in UK ${ }^{34}$ during the current COVID-19 emergency. This recommended rate will be accomplished by the FW configuration even if the bus moves at the very low speed of $9 \mathrm{~km} / \mathrm{h}$.

In the analysis we have left aside other variables that may be important in the design of urban buses such as the effects on the total bus hydrodynamic drag or other considerations such as the passengers comfort. In any case, our observations are useful to designers or engineers as it proposes a practical solution to an emergency situation in which the expulsion of aerosols becomes the relevant factor. On the other hand, while this study focus on the ventilation characteristics of the basic bus bodywork, further studies should be done to investigate the effects of temperature differences between the outer regions of the bus (different environmental conditions) and the inner temperature (passengers heat release) as well as the subsequent thermal plumes caused by these temperature differences ${ }^{35}$. As a note, the average inner velocity in the FW case was $0.8 m / s$ which is four times larger than the average velocity cause by the thermal plume as measured in stagnant conditions $(0.24 \mathrm{~m} / \mathrm{s}$ having $19.5^{\circ} \mathrm{C}$ in ambient air $^{36}$ and using a manikin); it is however of the same order of magnitude as the average velocity found in the $4 \mathrm{~W}$ case so it may impact the flow field in that case if the temperature differences are large.

We explored as well other open windows configurations that could, in principle, be attractive choices for the sake of improving the indoor ventilation. For instance, we constructed a geometry in which we modelled a driver's window (see the supplementary material S7). This window opens rotating a hinge so it can be oriented in a position of "attack" (windward) with respect to the outer flow. Surprisingly, we did not observe a notable improvement in terms of the rear-to-front pumping effect observed when two sets of windows pairs are open. Urban buses have also mobile gates at the roof, but they in principle do not offer a notable improvement or changes to the mean velocity structure compared to the traditional lateral windows (see Ref. ${ }^{18}$ for more discussion on this arrangement).

In connection with the turbulent models used in this work, we did not detect important differences between global expulsion rates of the aerosol cloud obtained with the $\kappa-\varepsilon$ or the SST $\kappa-\omega$ models, see figure 2 . As shown in the supplementary material $\mathrm{S} 4$, the flow field does present some differences depending on the turbulent model used. We also included a comparison between 

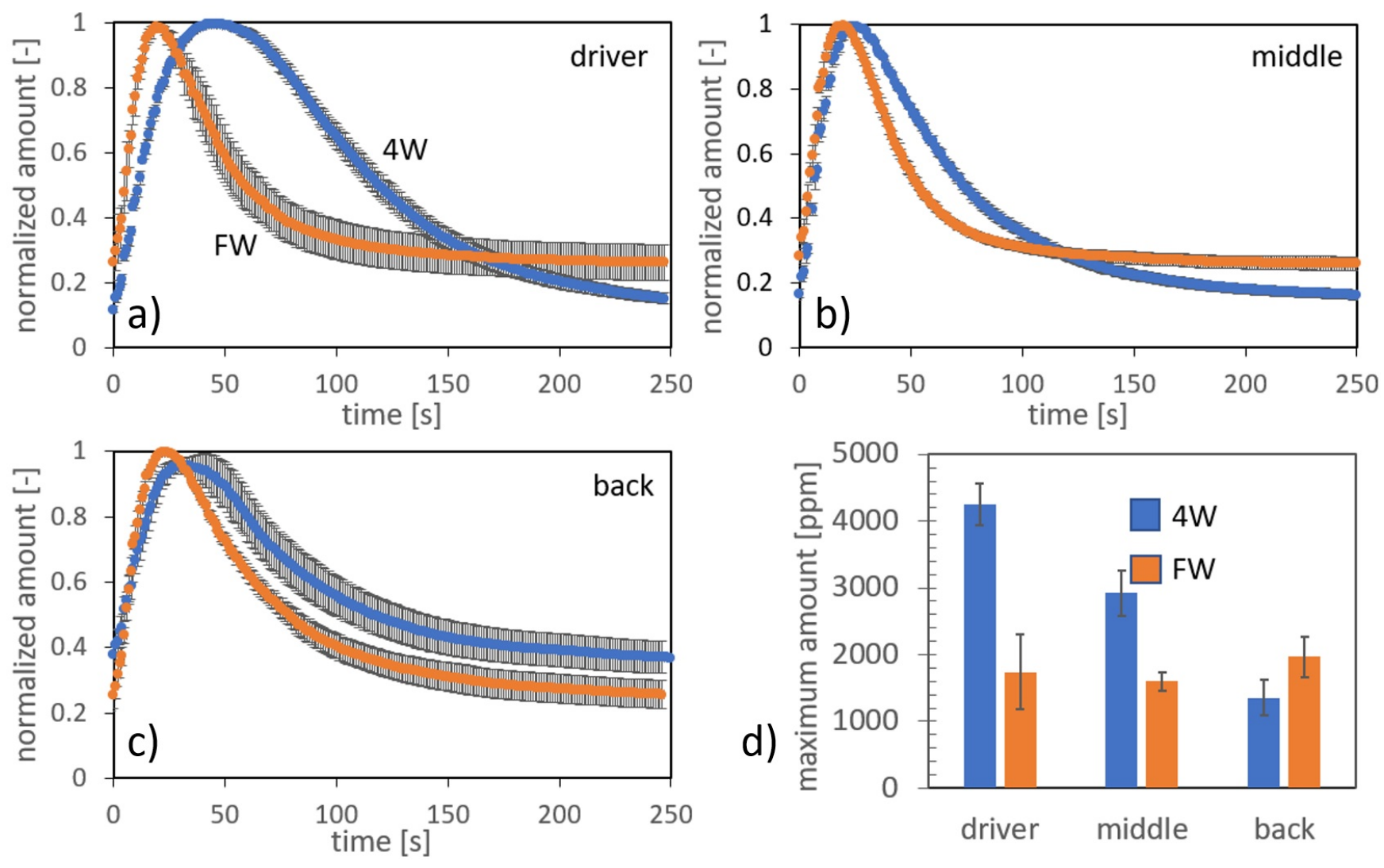

Figure 5. Ventilation experiments using $\mathrm{CO}_{2}$ as the passive tracer. (a), (b) and (c): $\mathrm{CO}_{2}$ concentration normalized with the maximum value at the emission peak as a function of time measured at the front (driver's area), middle and back areas of the bus, respectively, for the $4 \mathrm{~W}$ (blue line) and FW (orange line) configurations; (d): absolute $\mathrm{CO}_{2}$ concentration detected at the emission peak for each case. Error bars are indicated for 5 repetitions done in each condition.

the expulsion rates obtained with these two models but without including the turbulent mixing term in the diffusion-convection equation (see the methods section and S3). In that case, the difference in the expulsion rates is very notorious, underlining the fact that one should always include turbulent mixing in the governing mass transport equations for reliable results.

Finally, it is our hope that the present report can contribute to the general understanding of the physics and natural flows occurring inside urban buses and help decision makers to opt for design alternatives that can improve indoor ventilation in an emergency or critical situation. The current numerical results can also be used as a starting point for more advanced RANS models such as the Reynolds stress transport model, complemented with the so-called two-layer model and which have shown to improve particle deposition predictions ${ }^{37,38}$, or in Large Eddy Simulations or Direct Numerical Simulations which can provide more information about the turbulent and transport fluctuations of the indoor emitted aerosols.

\section{Methods}

\section{Numerical model and finite element specifications}

Figure 1 above shows a scheme of an urban bus together with the numerical and experimental models used in this report. In the numerical model we generated a real sized bus having length $(\mathrm{L})=9.92 \mathrm{~m}$, width $(\mathrm{W})=2.5 \mathrm{~m}$ and height $(\mathrm{H})=2.2 \mathrm{~m}$ leaving a gap of floor $=0.42 \mathrm{~m}$ between the internal floor of the bus and the ground level. Bus solid walls and internal seats were assumed to have zero thickness so that the bus bodywork is in fact a 2D numerical mesh embedded in a 3D numerical domain. The whole numerical bus was placed inside a 3D rectangular box of size $8 L \times 2 L \times 2 L$, see Figures 6 a and 6 b, putting the bus closer to the frontal external wall, $i$ (cartesian coordinates origin is placed at the frontal wall of the bus, with $y$-axis oriented with the bus length). The open windows are regions at the solid walls where the no-slip condition is not applied. The lateral windows have a size of $53 \times 40 \mathrm{~cm}$ while the frontal windows have $90 \times 20 \mathrm{~cm}$ each. A finer tetrahedral mesh was generated at the bus walls, interior, and zone immediately behind the bus rear wall where turbulent helical vortex structures are usually formed and which require more spatial resolution. ${ }^{39-41}$ A total of $1.3 \times 10^{6}$ elements were used in the $3 \mathrm{D}$ models and $5.9 \times 10^{4}$ in the $2 \mathrm{D}$ models 
(all the simulations were assumed to have left/right symmetry, except the AW case, so the number of elements only reflect half of the whole domain).

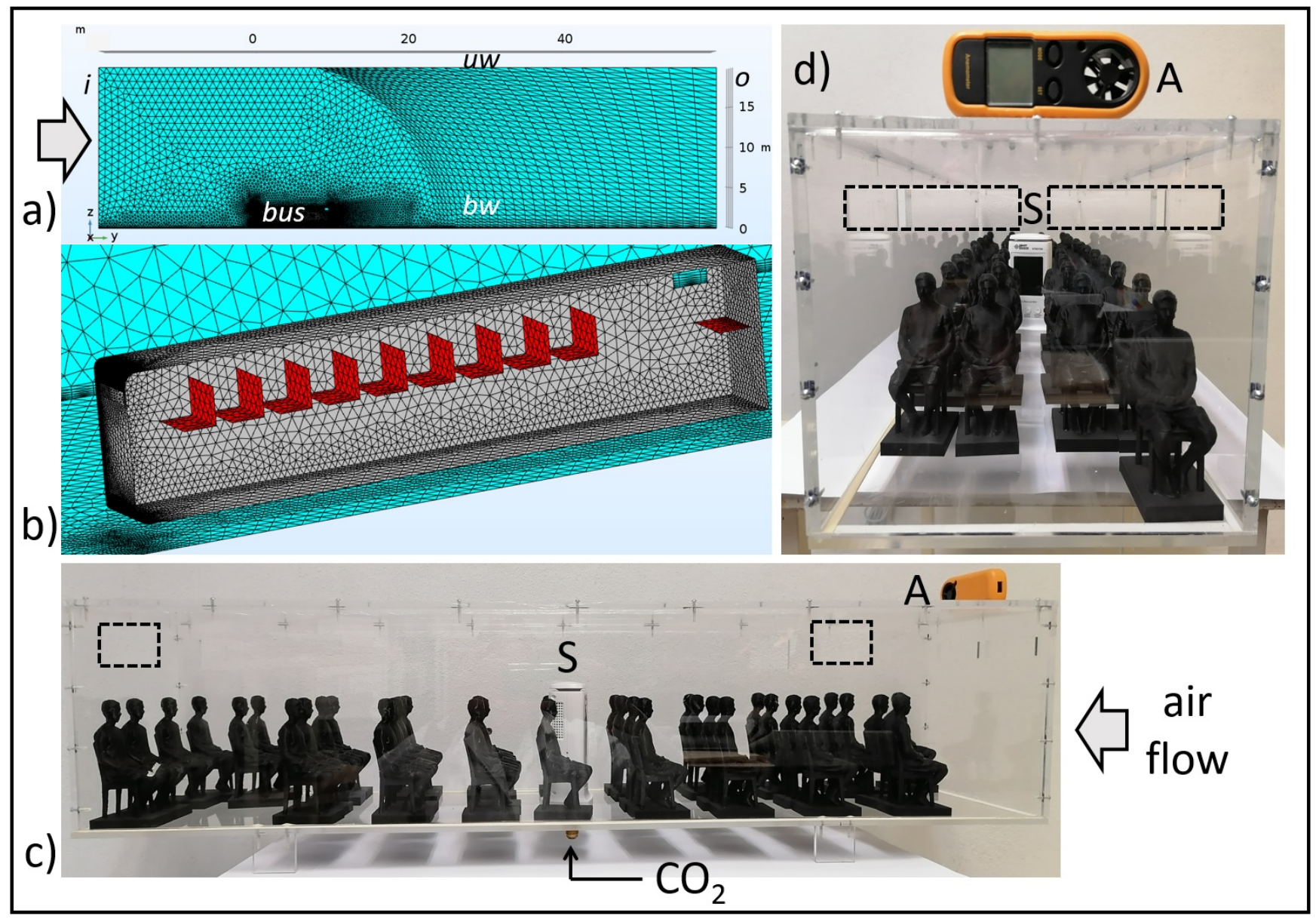

Figure 6. Numerical and experimental setups. (a) shows a panoramic view of the whole numerical domain, including the inlet wall (i) where air enters the domain, the outlet wall (o) and the upper wall (uw) which are considered open boundaries, and the bottom wall (bw) in which the no-slip condition is applied. The denser mesh region is where the bus is located; (b) zoom of the numerical domain showing the bus bodywork (gray) and the external walls (blue), the seats are marked in red; (c) and (d) show the acrylic bus model $(\mathrm{L}=1 \mathrm{~m})$, including the 3D-printed passenger figures, the $\mathrm{CO}_{2}$ feeding, $\mathrm{CO}_{2}$ sensor $(\mathrm{S})$ and the anemometer (A). The position of the windows are marked with dashed lines to guide the eye.

The simulations were ran as in a wind tunnel setup; i.e., the bus is fixed in space while the wind enters through the external wall " $i$ " shown in Figure 6 a at $U=50 \mathrm{~km} / \mathrm{h}$. The rest of the external walls "uw" and " $o$ " are assumed to be open boundaries (stress free condition), while at the bottom wall "bw" or ground level we applied the no-slip condition. The external Reynolds number was $R e \approx 2 \times 10^{6}$ based on the bus width. Simulations were ran in COMSOL Multiphysics.

\section{Fluid dynamics}

Air flow is assumed to be isothermal, incompressible and has no changes in the presence of aerosols (dilute regime ${ }^{13}$ ). In this way, we can decouple the fluid flow from the transport equations and solve them in two sequential steps. We then solved first the average steady velocity $\bar{u}_{j}$ and average pressure $\bar{p}$ following RANS models: ${ }^{42,43}$

$$
\nabla \cdot \bar{u}_{j}=0
$$

$$
\rho \bar{u}_{j} \frac{\partial \bar{u}_{i}}{\partial x_{j}}=-\frac{\partial \bar{p}}{\partial x_{i}}+\frac{\partial}{\partial x_{j}}\left[\left(\mu+\mu_{T}\right) \frac{\partial \bar{u}_{i}}{\partial x_{j}}\right]
$$


where $\rho$ and $\mu$ are the medium density and viscosity, respectively, while $\mu_{T}$ is the turbulent viscosity obtained either with the $\kappa-\varepsilon$ or the SST $\kappa-\omega$ turbulent models. Whenever we tried to find a convergent solution with SST $\kappa-\omega$ we started with $\kappa-\varepsilon$ to find a first solution. Standard wall functions were applied to $\kappa-\varepsilon$ on the walls having no-slip condition, while the Wolfshtein wall model ${ }^{44}$ was applied to the SST $\kappa-\omega$. On the other hand, since both models need to solve for the turbulent kinetic energy $\kappa$, we fed the model with values for $\kappa$ applied on the inlet wall " $i$ " (see Fig. 6a) obtained from wind tunnel experiments. ${ }^{40}$ In the supplementary material we have provided more references regarding previous validations and tests done to the SST $\kappa-\omega$ model.

\section{Diffusion-convection equation}

Once we solved for the average fluid flow in steady state, we then proceeded with the aerosol transport simulations using two different approaches. In the first one we considered a concentration-based approach (continuous formulation) of the aerosol cloud, where $\bar{c}$ represents the average concentration field and whose governing equation can be written as:

$$
\frac{\partial \bar{c}}{\partial t}+\bar{u}_{j} \frac{\partial \bar{c}}{\partial x_{j}}=\frac{\partial}{\partial x_{j}}\left[\Gamma_{c} \frac{\partial \bar{c}}{\partial x_{j}}\right]+S\left(x_{o}, t\right),
$$

where

$$
\Gamma_{c}=\mathscr{D}+\mathscr{D}_{T}=\mathscr{D}+\frac{\mu_{T}(\vec{x})}{\rho S c}
$$

being $\mathscr{D}$ the mass diffusion coefficient and $\mathscr{D}_{T}$ the turbulent diffusion or turbulent mixing. Additionally, $S c$ is the turbulent Schmidt number whose value is close to a constant, $\sim 0.7$, for a wide range of Re numbers, ${ }^{45}$ but can alternatively be defined in terms of the flow field variables ${ }^{46}$. To consider the emission of aerosols beginning at time $t=0$, we have included a source term in Eq.3 which has the form $S\left(x_{o}, t\right)=\dot{q}$ pulse $\left.(t) \hat{c}\right|_{\text {node: } x_{o}}$. Here pulse $(t)$ is a smooth pulse function with a standard deviation of $0.5 \mathrm{~s}, \hat{c}$ is the test function for the concentration field in the finite element formalism and $\dot{q}$ is the strength of the emission occurring at the node $x_{o}$. As a case of study, the point source is placed at $x_{o}=\{ \pm 0.6,5,1.6+$ floor $\}[\mathrm{m}]$. Since we are assuming that the aerosols are so diluted that they do not change the hydrodynamic properties of air, in practice $\dot{q}$ can have any value; therefore, we present the evolution of $\bar{c}(x, t)$ normalized with the maximum value obtained at the peak of the pulse. In fact, in Figure 2 we showed the total amount of aerosols, $\left.\mathscr{N}(t)\right|_{\text {in }}$, inside the bus at any time after emission being normalized with the maximum value appearing at time $t_{\max }$, i.e.,

$$
\left.\mathscr{N}(t)\right|_{\text {in }}=\frac{\int_{\text {in }} c(x, t) d \mathscr{V}}{\int_{\text {in }} c\left(x, t_{\text {max }}\right) d \mathscr{V}}
$$

\section{Lagrangian formulation of aerosols}

The aerosol cloud was also modelled explicitly by following a set of Lagrangian spherical particles emitted at the same site $x_{o}$ as in the continuous formulation. Newton's equations were solved by considering the drag force $F_{d}$ as follows:

$$
m_{p} \frac{d u_{p, i}}{d t}=\vec{F}_{d}=\frac{m_{p}}{C_{c}} \frac{3 \mu C_{D} R e_{r}}{4 \rho_{p} d_{p}^{2}}\left(\bar{u}_{i}-u_{p, i}\right)
$$

where $m_{p}, \rho_{p}, d_{p}$ and $\vec{u}_{p}$ are the mass, density, diameter and velocity of the particles while $\bar{u}_{i}$ is the average steady velocity obtained with the RANS models. Here we are interested in following the path of aerosol particles emitted during normal breathing; therefore, we considered the particle distribution found experimentally in Ref. ${ }^{47}$ and whose particle size range is 0.7 to $5.5 \mu \mathrm{m}$ with peak at $1.5 \mu \mathrm{m}$ (we considered only probability densities higher than $10^{-2}$, smaller particle size fractions were neglected). For these particles sizes buoyancy forces can be neglected $\left(\rho_{p}=1000 \mathrm{~kg} / \mathrm{m}^{3}\right)$ as well as evaporation dynamics due to its small characteristic time scale. ${ }^{30}$ On the other hand, with these particle sizes the Knudsen number is not so small, $K n \sim 0.1$, so the Cunningham slip correction ${ }^{48}$ is applied to the drag force as:

$$
C_{c}(K n)=1+K n[2.514+0.8 \exp \{-0.55 / K n\}]
$$

although we did not detect significant changes when $C_{c}$ was not included. The drag factor $C_{D}\left(\operatorname{Re}_{r}\right)$ was calculated using standard tables valid up to $\operatorname{Re}_{r} \sim 10^{6}$, although the results are similar if the Schiller-Naumann ${ }^{49}$ empirical formula is used instead. Here the relative Reynolds number is defined as $R e_{r}=\rho\left|\vec{u}-\vec{u}_{p}\right| d_{p} / \mu$. To simulate the exhalation event we released 100 particles at $x_{o}$ for a time window of 2 s having a uniform distribution of unit vectors coming out from a $47^{\circ}$ cone ${ }^{50}$ with $z$-axis oriented to the front of the bus. In order to account for the randomness nature of the aerosol distribution in an exhalation, we repeated the exhalation event 40 times, each time picking different delivery times for each particle within the $2 \mathrm{~s}$ window 
and different speeds or velocity magnitudes using a random generator number. The exhalation speed range was 0 to $20 \mathrm{~m} / \mathrm{s}$, being the upper limit the characteristic velocity of a cough. ${ }^{51}$ As an additional note, taking into account the Brownian motion expressed in terms proportional to $\sim \sqrt{k_{B} T}$ did not make substantial difference in the simulations. Finally, the sticky condition was applied to the particles whenever they touch a no-slip surface, i.e., they become arrested.

\section{Mean age of air}

While the common transport equations (Lagrangian or Eulerian) allow us to see explicitly the drag of aerosols and its subsequent expulsion through the open windows, their application is somehow limited because each chosen point of emission will experience a different hydrodynamic drag depending on its particular location; in order words, we will have to probe a considerable number of different point sources in order to have a complete picture of the retention time (or expulsion rate) of the aerosols and detect regions with poor ventilation. Therefore, in order to reach a more general picture of the ventilation quality inside the bus, we also computed the so-called mean age of air, $\mathscr{A}^{52}$, in the outer and inner regions. The interpretation of $\mathscr{A}$ may be given in the following terms: imagine that the bus receives, at a certain time $t=0$, a bundle of particles moving with the incoming flow from wall " $i$ " (Fig. 6a); then, at some location in or outside the bus, an observer detects such particles when they pass through that specific location; the scalar field $\mathscr{A}$ will be then the average time that has past since the release of the particles at the inlet until the observer detects the passage of the particle bundle. Formally, the local value of $\mathscr{A}$ is defined as:

$$
\mathscr{A}(x)=\frac{\int_{0}^{\infty} t c(x, t) d t}{\int_{0}^{\infty} c(x, t) d t}
$$

As shown by Li and Tilton, Sandberg and Spalding ${ }^{52-54}$, the transport equation 3 can be manipulated in order to accommodate the definition given by 8 to finally obtain a partial differential equation for $\mathscr{A}$ in the form:

$$
\nabla \cdot\left(\bar{u}_{i} \mathscr{A}\right)=\nabla \cdot\left[\Gamma_{c} \nabla \mathscr{A}\right]+1
$$

As commented by Liu and Tilton, equation 9 has the same form as the steady transport equation for diluted species with an extra term corresponding to a reaction term equal to 1 . In this sense, we can solve the field values of $\mathscr{A}$ using the mean velocities given by the turbulent models and using the same numerical solvers used for regular PDEs. In the supplementary material we have included a validation test for $\mathscr{A}$ and which shows that its value at the outlet wall "o" (see Fig. 6a) is in good agreement with the total time the air spends in travelling the whole computational domain, i.e., $8 L / U=79.3[\mathrm{~m}] / 13.9[\mathrm{~m} / \mathrm{s}]=5.7 \mathrm{~s}$. Finally, for the field $\mathscr{A}$ we applied the Dirichlet condition $\mathscr{A}=0$ at the inlet " $i$ ", while in the rest of the walls, including the solid walls and outlets, we applied the Neumann condition $\vec{n} \cdot \nabla \mathscr{A}=0$.

\section{Experimental validation}

Ventilation experiments were performed using a 1:10 scale model of an urban bus made of acrylic and 3D-printed figures of seated people, see Figures 1c, 6c and 6d. As the scaling parameter we opted to fix the time it takes the air stream to travel the full bus length, i.e., $\sim 0.7 \mathrm{~s}$. An anemometer (A) was used to adjust the air speed, $\sim 1.3 \mathrm{~m} / \mathrm{s}$, as provided by a regular fan placed in front of the model. We used $\mathrm{CO}_{2}$ as a passive tracer to infer the ventilation characteristics given a distribution of open windows (the windows were simply holes made on the acrylic walls and closed if necessary with acetate). Pulses of $\mathrm{CO}_{2} 0.5 \mathrm{~s}$ long were introduced inside the bus through a connection placed at the middle of the bus (see Fig. 6c). A wireless commercial $\mathrm{CO}_{2}$ sensor (S) and recorder (ST8310A, 0-20,000 ppm) was placed at the middle, back and front positions to follow the $\mathrm{CO}_{2}$ concentration inside the model after release at time $t=0$. Five repetitions were done for each condition, detaching the upper cap of the model between consecutive measurements to dissipate the remaining $\mathrm{CO}_{2}$.

\section{References}

1. Morawska, L. et al. How can airborne transmission of covid-19 indoors be minimised? Environ. Int. 142, 105832-7 (2020).

2. Lewis, D. Mounting evidence suggests coronavirus is airborne — but health advice has not caught up. Nature 583, 510-513, DOI: 10.1038/d41586-020-02058-1 (2020).

3. Tang, S. et al. Aerosol transmission of sars-cov-2? evidence, prevention and control. Environ. Int 144, 106039-10, DOI: doi.org/10.1016/j.envint.2020.106039 (2020).

4. Milton, D. K., Fabian, M. P., Cowling, B. J., Grantham, M. L. \& McDevitt, J. J. Influenza virus aerosols in human exhaled breath: Particle size, culturability, and effect of surgical masks. PLOS Pathog. 9, e1003205-8, DOI: 10.1371/journal.ppat. 1003205 (2013). 
5. Stadnytskyi, V., Bax, C., Bax, A. \& Anfinrud, P. The airborne lifetime of small speech droplets and their potential importance in sars-cov-2 transmission. PNAS 117, 11875-11877 (2020).

6. Jayaweera, M., Perera, H., Gunawardana, B. \& Manatunge, J. Transmission of covid-19 virus by droplets and aerosols: A critical review on the unresolved dichotomy. Environ. Res. 118, 109819-18 (2020).

7. Somsen, G., van Rijn, C., Kooij, S., Bem, R. \& Bonn, D. Small droplet aerosols in poorly ventilated spaces and sars-cov-2 transmission. Lancet Respir. Med. 8, 658-659 (2020).

8. Zhang, Z. et al. Disease transmission through expiratory aerosols on an urban bus. Phys. Fluids 33, 015116-16 (2021).

9. Port, J. R. et al. Increased small particle aerosol transmission of b.1.1.7 compared with sars-cov-2 lineage a in vivo. Nat. Microbiol. DOI: 10.1038/s41564-021-01047-y (2022).

10. Prather, K. A. et al. Airborne transmission of sars-cov-2. Science 370, 303-304 (2020).

11. Abulhassan, Y. \& (Jerry), G. A. D. Considerations for the transportation of school aged children amid the coronavirus pandemic. Transp. Res. Interdiscip. Perspectives 9, 100290-5 (2021).

12. Browne, A., Ahmad, S. S.-O., Beck, C. R. \& Nguyen-Van-Tam, J. S. The roles of transportation and transportation hubs in the propagation of influenza and coronaviruses: a systematic review. J. Travel. Med. 23, 1-7, DOI: 10.1093/jtm/tav002 (2016).

13. Yang, X. et al. Transmission of pathogen-laden expiratory droplets in a coach bus. J. Hazard Mater. 397, 122609-15, DOI: 10.1016/j.jhazmat.2020.122609 (2020).

14. Zhu, S., Srebric, J., Spengler, J. D. \& Demokritou, P. An advanced numerical model for the assessment of airborne transmission of influenza in bus microenvironments. Build Environ. 47, 67-75, DOI: 10.1016/j.buildenv.2011.05.003 (2012).

15. Zhu, S., Philip, D. \& Spengler, J. D. Experimental and numerical investigation of micro-environmental conditions in public transportation buses. Build Environ. 45, 2077-2088 (2010).

16. Li, F., Lee, E. S., Zhou, B., Liu, J. \& Zhu, Y. Effects of the window openings on the micro-environmental condition in a school bus. Atmos. Environ. 167, 434-443, DOI: 10.1016/j.atmosenv.2017.08.053 (2017).

17. Kale, S., Veeravalli, S., Punekar, H. \& Yelmule, M. Air flow through a non-airconditioned bus with open windows. Sadhana 32, 347-363, DOI: 10.1007/s12046-007-0029-3 (2007).

18. Xingjun, H., Fengtao, R., Bo, Y. \& Peng, G. Effect of sunroofs and side windows on aerodynamic characteristics of transit bus. Appl. Mech. Mater. 224, 333-337, DOI: 10.4028/www.scientific.net/AMM.224.333 (2012).

19. Lin, B., Wang, X. \& Hu, X. Research on the effect of natural ventilation on buses in summer based on cfd numerical simulation method. Adv. Mat. Res. 361, 1056-1060, DOI: 10.4028/www.scientific.net/AMR.361-363.1056 (2012).

20. Lee, E. S., Fung, C.-C. \& Zhu, Y. Evaluation of a high efficiency cabin air (heca) filtration system for reducing particulate pollutants inside school buses. Environ. Sci. Technol. 49, 3358-3365 (2015).

21. Li, F., Lee, E. S., Liu, J. \& Zhu, Y. Predicting self-pollution inside school buses using a cfd and multi-zone coupled model. Atmos. Environ. 107, 16-23 (2015).

22. Mesgarpour, M. et al. Prediction of the spread of corona-virus carrying droplets in a bus - a computational based artificial intelligence approach. J. Hazard Mater. 413, 125358-18, DOI: 10.1016/j.jhazmat.2021.125358 (2021).

23. Li, F., Liu, J., Ren, J., Cao, X. \& Zhu, Y. Numerical investigation of airborne contaminant transport under different vortex structures in the aircraft cabin. Int. J. Heat Mass Transf. 96, 287-295, DOI: 10.1016/j.ijheatmasstransfer.2016.01.004 (2016).

24. Zee, M. et al. Computational fluid dynamics modeling of cough transport in an aircraft cabin. Sci. Rep. 11, 23329-10, DOI: 10.1038/s41598-021-02663-8 (2021).

25. Li, N. et al. Multi-objective optimization for designing of high-speed train cabin ventilation system using particle swarm optimization and multi-fidelity kriging. Build Environ. 155, 161-174, DOI: 10.1016/j.buildenv.2019.03.021 (2019).

26. Mathai, V., Das, A., Bailey, J. A. \& Breuer, K. Airflows inside passenger cars and implications for airborne disease transmission. Sci. Adv. 7, eabe0166-7, DOI: 10.1126/sciadv.abe0166 (2021).

27. Zhang, Y., Feng, G., Kang, Z., Bi, Y. \& Cai, Y. Numerical simulation of coughed droplets in conference room. Procedia Eng. 205, 302-308, DOI: 10.1016/j.proeng.2017.09.981 (2017). 
28. Issakhov, A. et al. A numerical assessment of social distancing of preventing airborne transmission of covid-19 during different breathing and coughing processes. Sci. Rep. 11, 9412-39, DOI: 10.1038/s41598-021-88645-2 (2021).

29. Vuorinen, V. et al. Modelling aerosol transport and virus exposure with numerical simulations in relation to sars-cov-2 transmission by inhalation indoors. Saf. Sci. 130, 104866-43, DOI: 10.1016/j.ssci.2020.104866 (2020).

30. Liu, H., He, S., Shen, L. \& Hong, J. Simulation-based study of covid-19 outbreak associated with air-conditioning in a restaurant. Phys. Fluids 33, 023301-18, DOI: doi.org/10.1063/5.0040188 (2021).

31. Peng, S., Chen, Q. \& Liu, E. The role of computational fluid dynamics tools on investigation of pathogen transmission: Prevention and control. Sci. Total. Environ. 746, 142090-23, DOI: 10.1016/j.scitotenv.2020.142090 (2020).

32. Sun, C. \& (John), Z. Z. The efficacy of social distance and ventilation effectiveness in preventing covid-19 transmission. Sustain. Cities Soc. 62, 102390-10, DOI: 10.1016/j.scs.2020.102390 (2020).

33. Ponte, C. et al. Tracing contacts to evaluate the transmission of covid-19 from highly exposed individuals in public transportation. Sci. Rep. 11, 24443-11, DOI: 10.1038/s41598-021-03998-y (2021).

34. Tirachini, A. \& Cats, O. Covid-19 and public transportation: Current assessment, prospects, and research needs. J. Public Transp. 22, 1-21, DOI: 10.5038/2375-091.22.1.1 (2020).

35. Yan, Y., Li, X. \& Tu, J. Effects of passenger thermal plume on the transport and distribution characteristics of airborne particles in an airliner cabin section. Sci. Technol. Built En. 22, 153-163, DOI: doi.org/10.1080/23744731.2015.1090254 (2016).

36. Wang, C., Liu, J., Li, J. \& Li, F. Chaotic behavior of human thermal plumes in an aircraft cabin mockup. Int. J. Heat Mass Tran. 119, 223-235, DOI: 10.1016/j.ijheatmasstransfer.2017.11.059 (2018).

37. Tian, L. \& Ahmadi, G. Particle deposition in turbulent duct flows—comparisons of different model predictions. J. Aerosol Sci. 38, 377-397, DOI: 10.1016/j.jaerosci.2006.12.003 (2007).

38. Chen, H. C. \& Patel, V. C. Near-wall turbulence models for complex flows including separation. AIAA J. 26, 641-648, DOI: $10.2514 / 3.9948$ (1988).

39. Menter, F. \& Kuntz, M. Adaptation of Eddy-Viscosity Turbulence Models to Unsteady Separated Flow Behind Vehicles, In: McCallen R., Browand F., Ross J. (eds) "The Aerodynamics of Heavy Vehicles: Trucks, Buses, and Trains", Lecture Notes in Applied and Computational Mechanics (Springer-Verlag, Berlin, 2004).

40. Lienhart, H. \& Becker, S. Flow and turbulence structure in the wake of a simplified car model. SAE Tech. Pap. Ser. 2003-01-0656, 1-12 (2003).

41. Jakirlić, S., Jester-Zücker, R. \& Tropea, C. 9th ERCOFTAC/IAHR/COST Workshop on Refined Turbulence modelling (Darmstadt University of Technology, Germany, 2001).

42. Davidson, P. A. turbulence; An introduction for scientists and engineers (Oxford University Press, India, 2015), 2nd edition edn.

43. Zhang, Z., Zhang, W., Zhai, Z. J. \& Chen, Q. Y. Evaluation of various turbulence models in predicting airflow and turbulence in enclosed environments by cfd: Part 2-comparison with experimental data from literature. HVAC\&R Res. 13:6, 871-886 (2007).

44. Wolfshtein, M. The velocity and temperature distribution in one-dimensional flow with turbulence augmentation and pressure gradient. Int. J. Heat Mass Transf. 12, 301-318 (1969).

45. Goldman, I. B. \& Marchello, J. M. Turbulent schmidt numbers. Int. J Heat Mass Transf. 12, 797-802 (1969).

46. Li, F., Liu, J., Ren, J. \& Cao, X. Predicting contaminant dispersion using modified turbulent schmidt numbers from different vortex structures. Build Environ. 130, 120-127 (2018).

47. Shao, S. et al. Risk assessment of airborne transmission of covid-19 by asymptomatic individuals under different practical settings. J. Aerosol Sci. 151, 105661-9, DOI: 10.1016/j.jaerosci.2020.105661 (2021).

48. Buckley, R. \& S.K.Loyalka. Cunningham correction factor and accommodation coefficient: Interpretation of millikan's data. J. Aerosol Sci. 20, 347-349, DOI: 10.1016/0021-8502(89)90009-8 (1989).

49. Homann, H., Bec, J. \& Grauer, R. Effect of turbulent fluctuations on the drag and lift forces on a towed sphere and its boundary layer. J. Fluid Mech. 721, 155-179, DOI: 10.1017/jfm.2013.66 (2013).

50. Xua, C., Nielsen, P. V., Liu, L., L.Jensen, R. \& Gong, G. Human exhalation characterization with the aid of schlieren imaging technique. Build Environ. 112, 190-199, DOI: 10.1016/j.buildenv.2016.11.032 (2017). 
51. Kwon, S.-B. et al. Study on the initial velocity distribution of exhaled air from coughing and speaking. Chemosphere 87, 1260-1264, DOI: 10.1016/j.chemosphere.2012.01.032 (2012).

52. Liu, M. \& Tilton, J. N. Spatial distributions of mean age and higher moments in steady continuous flows. AICHE J. 56, 2561-2572 (2010).

53. Sandberg, M. What is ventilation efficiency? Build Environ. 16, 123-135 (1981).

54. Spalding, D. B. A note on mean residence-times in steady flows of arbitrary complexity. Chem. Eng. Sci. 9, 74-77 (1958).

\section{Acknowledgements}

The authors acknowledge the Investigadores por México program and CONACyT grant FORDECYT-PRONACES/731759/2020. We also aknowledge the support of Laboratorio Nacional de la Materia fuera de Equilibrio, LANIMFE, and of Jesús Israel García Villaseñor for the model construction.

\section{Author contributions statement}

F.A.P.O. made the experiments and simulations, O.A.P.L. made the experiments, J.R.V.C. made simulations and wrote the paper.

\section{Competing interests}

Te authors declare no competing interests.

\section{Data availability}

All data generated or analysed during this study are included in this published article [and its supplementary information files]. 


\section{Supplementary Files}

This is a list of supplementary files associated with this preprint. Click to download.

- SUPPLEMENTARYMATBUS.pdf

- Windowsmovies.wmv 